\title{
MMR Deficiency is Homogeneous in Pancreatic Carcinoma and Associated with High Density of Cd8-Positive Lymphocytes
}

\author{
Christoph Fraune, MD ${ }^{1}$, Eike Burandt, $\mathrm{MD}^{1}$, Ronald Simon, $\mathrm{PhD}^{\mathbf{1}}$, Claudia Hube-Magg, $\mathrm{PhD}^{\mathbf{1}}$, \\ Georgia Makrypidi-Fraune, $\mathbf{P h D}^{1}$, Martina Kluth, $\mathbf{P h D}^{1}$, Franziska Büscheck, $\mathbf{M D}^{1}$, Doris Höflmayer, $\mathbf{M D}^{1}$, \\ Niclas Ch. Blessin ${ }^{1}$, Tim Mandelkow', Wenchao $\mathrm{Li}^{1}$, Daniel Perez, $\mathrm{MD}^{2}$, Jakob R. Izbicki, $\mathrm{MD}^{2}$, \\ Waldemar Wilczak, MD ${ }^{1}$, Guido Sauter, MD ${ }^{1}$, Jörg Schrader, MD $^{2,3}$, Michael Neipp, MD $^{4}$, \\ Hamid Mofid, $\mathrm{MD}^{5}$, Thies Daniels, $\mathrm{MD}^{6}$, Christoph Isbert, $\mathrm{MD}^{7}$, Till S. Clauditz, MD ${ }^{1}$, and \\ Stefan Steurer, MD $^{1}$
}

${ }^{1}$ Institute of Pathology, University Medical Center Hamburg-Eppendorf, Hamburg, Germany; ${ }^{2}$ General, Visceral and Thoracic Surgery Department and Clinic, University Medical Center Hamburg-Eppendorf, Hamburg, Germany; ${ }^{3}$ I. Medical Department - Gastroenterology and Hepatology, University Medical Center Hamburg-Eppendorf, Hamburg, Germany; ${ }^{4}$ General, Vascular and Visceral Surgery Clinic, Itzehoe Medical Center, Itzehoe, Germany; ${ }^{5}$ General, Visceral Thoracic and Vascular Surgery Clinic, Regio Clinic Pinneberg, Pinneberg, Germany; ${ }^{6}$ General, Visceral and Tumor Sugery Clinic, Albertinen Hospital, Hamburg, Germany; ${ }^{7}$ Department of General, Gastrointestinal and Colorectal Surgery, Amalie Sieveking Hospital, Hamburg, Germany

\begin{abstract}
Background. Microsatellite instability (MSI) has emerged as a predictive biomarker for immune checkpoint inhibitor therapy. Cancer heterogeneity represents a potential obstacle for the analysis of predicitive biomarkers. MSI has been reported in pancreatic cancer, but data on the possible extent of intratumoral heterogeneity are lacking.

Methods. To study MSI heterogeneity in pancreatic cancer, a tissue microarray (TMA) comprising 597 tumors was screened by immunohistochemistry with antibodies for the mismatch repair (MMR) proteins MLH1, PMS2, MSH2, and MSH6.

Results. In six suspicious cases, large section immunohistochemistry and microsatellite analysis (Bethesda panel) resulted in the identification of $4(0.8 \%)$ validated MSI cases out of 480 interpretable pancreatic ductal
\end{abstract}

Christoph Fraune and Eike Burandt have equally contributed to the work described in this manuscript

(C) The Author(s) 2020, corrected publication 2022

First Received: 29 October 2019

R. Simon, $\mathrm{PhD}$

e-mail: R.Simon@uke.de adenocarcinomas. MSI was absent in 55 adenocarcinomas of the ampulla of Vater and 7 acinar cell carcinomas. MMR deficiency always involved MSH6 loss, in three cases with additional loss of MSH2 expression. Three cancers were MSI-high and one case with isolated MSH6 loss was MSS in PCR analysis. The analysis of 44 cancer-containing tumor blocks revealed that the loss of MMR protein expression was always homogeneous in affected tumors. Automated digital image analysis of CD8 immunostaining demonstrated markedly higher CD8 + tumor infiltrating lymphocytes in tumors with $($ mean $=685$, median $=626)$ than without $($ mean $=227$; median $=124)$ MMR deficiency $(p<0.0001)$, suggesting a role of MSI for immune response.

Conclusions. Our data suggest that MSI occurs early in a small subset of ductal adenocarcinomas of the pancreas and that immunohistochemical MMR analysis on limited biopsy or cytology material may be sufficient to estimate MMR status of the entire cancer mass.

Despite recent progress in cancer therapy, pancreatic cancer-with more than 55,000 new cases diagnosed in the United States annually-still confers dismal prognosis. ${ }^{1}$ Due to the lack of early symptoms, late diagnosis, early metastatic dissemination, and ineffective systemic 
therapies, prognosis for these tumors is poor. Although pancreatectomy can prolong life in eligible patients, less than $5 \%$ of patients will survive more than 5 years. Immune checkpoint inhibitors have become a breakthrough treatment modality for many solid cancers but initially showed little effect in pancreatic cancer. ${ }^{2,3}$ However, based on data showing strong favorable responses of microsatellite instable (MSI) tumors-independent of the site of tumor origin - to the PD-1 antibody pembrolizumab, this drug obtained a site-agnostic FDA approval for treatment of advanced cancers with MMR deficiency/MSIhigh. ${ }^{4,5}$ As several MSI pancreatic cancers had responded well to pembrolizumab in a recent study, the MSI status of pancreatic cancers is increasingly gaining interest. ${ }^{6}$

MSI reflects a hypermutator phenotype inducing a high mutational load in affected cancers and is typically caused by a deficient mismatch repair (MMR) system unable to resolve short slippage DNA errors that occur during cell cycle. It can be detected directly with polymerase chain reaction (PCR)-based methods demonstrating variable allele length of microsatellites or indirectly by identifying an expression loss of the MMR proteins MLH1, PMS2, MSH2, or MSH6 by immunohistochemistry (IHC). MSI occurs in various malignancies. Highest frequencies have been reported for endometrial (21-30\%), colon (up to $19 \%$ ), and stomach cancer (6-22\%), but an increasing number of studies is showing that MSI can be found in virtually all individual cancer types at a frequency of approximately $1 \% .^{7-15}$ Tumor-infiltrating lymphocytes have been linked to tumor phenotype as well as favorable patient outcome or response to therapy in various tumor types. ${ }^{16-18}$ MSI is associated with a high number of tumorinfiltrating lymphocytes providing indirect evidence for a particular role of the antitumoral immune response in such tumors, presumably due to increased neoantigen production. ${ }^{19-21}$

Reports on MSI in pancreatic cancer described variable results. MSI has been reported to occur in $0-22 \%$ of unselected cases when IHC was used to assess MMR deficiency and in $0-17 \%$ in reports utilizing PCR based methods. ${ }^{22-25}$ Given the pivotal impact on the selection of treatment, precise assessment of MSI is important. Especially in those pancreatic cancers that are not amenable to surgery, therapeutically relevant molecular information is generally obtained from biopsies or cytological specimen. Such a small fraction of the primary tumor may not always be reflective of the molecular status of the entire cancer mass. Tumor heterogeneity can confound molecular diagnostics and diminish the success of targeted therapies. Intratumoral heterogeneity of MMR status has been described in some cases of colorectal and endometrial cancer but has so far not been analyzed in pancreatic adenocarcinoma. ${ }^{26-30}$
To learn more on MSI heterogeneity in pancreatic cancer, a cohort of 597 operated pancreatic cancers was screened by IHC for loss of the MMR proteins MLH1, PMS2, MSH2, and/or MSH6 on a tissue microarray (TMA). Cases with suspected MSI were further analyzed by PCR and repeated IHC on large sections followed by a thorough analysis of all available cancer-containing tissue blocks for possible intratumoral heterogeneity. Moreover, the relationship of MSI with the number of CD8-positive tumor-infiltrating lymphocytes was analyzed to assess the biologic impact of MSI in pancreatic cancers.

\section{MATERIALS AND METHODS}

\section{Tissue Microarray}

A tissue microarray was constructed from a consecutive series of 597 pancreatic carcinomas treated by pancreatectomy ( $\mathrm{pT} \geq 2$ ), of which the specimens were analyzed at the Institute of Pathology of the University Medical Center Hamburg-Eppendorf. The series included 529 pancreatic ductal adenocarcinomas, 61 adenocarcinomas of the ampulla of Vater, and 7 acinar cell carcinomas. Data on pT and $\mathrm{pN}$ category, histological grade, tumor diameter, and presence or absence of distant metastasis were taken from the pathology reports. TMA construction was done as described. ${ }^{31}$ In brief, tissue cylinders with a diameter of $0.6 \mathrm{~mm}$ each were taken from representative tumor areas of selected tumor "donor" tissue blocks using a homemade semiautomated precision instrument and brought into empty recipient paraffin blocks. Utilization of archived diagnostic leftover tissues for manufacturing of tissue microarrays and their analysis for research purposes as well as patient data analysis has been approved by local laws (HmbKHG, §12,1) and by the local ethics committee (Ethics commission Hamburg, WF-049/09). All work has been performed in compliance with the Helsinki Declaration.

\section{Immunohistochemical Analyses}

Freshly taken TMA sections were used for MMR protein analysis in an automated immunostainer (Dako/Agilent Autostainer Link 48). Primary antibody specific for MLH1 (clone ES05, mouse), PMS2 (clone EP51, rabbit), MSH2 (clone FE11, mouse), and MSH6 (clone EP49, rabbit) (all Ready-to-Use, all from DAKO, Glostrup, Denmark) was applied for $20 \mathrm{~min}$ (MLH1, MSH2, MSH6) or $30 \mathrm{~min}$ (PMS2). A manual approach was used for CD8 immunostaining. Deparaffinized slides were exposed to a heat-induced antigen retrieval procedure for $5 \mathrm{~min}$ in an autoclave at $121^{\circ} \mathrm{C}$ in $\mathrm{pH}$ 7,8 Tris-EDTA-Citrate buffer. 
Primary antibody specific for CD8 (Oncodianova, mouse monoclonal antibody, Clone TC8, 1:450) was applied at $37{ }^{\circ} \mathrm{C}$ for $60 \mathrm{~min}$. Bound antibody was visualized using the EnVision Kit (Dako, Glostrup, Denmark) according to the manufacturer's directions. Nuclear MMR protein staining intensity was scored as $0,1+, 2+$, or $3+$ in cancer cells and the fraction (percentage) of stained tumor cells was also recorded for each tissue spot. In spots showing a negative (0) result for the tumor cells, presence $(+)$ or absence $(-)$ of nuclear staining in peritumoral stromal or inflammatory cells was additionally recorded as an internal control. For TMA spots with suspected MSI, IHC was repeated on a large section of the routinely archived tumor material. In case of confirmed MMR deficiency/MSI, all available archived tumor-containing blocks also were analyzed by IHC.

\section{PCR Analysis}

For all cases with suspected MMR deficiency based on TMA screening, fluorescent PCR-based assay (MSI Analysis System; Promega, Madison, WI) was used to analyze five microsatellite loci, including the two mononucleotide repeats (BAT25, BAT26) and three dinucleotide repeats (D2S123, D5S346, and D17S250) of the "BethesdaPanel." Analysis was based on DNA extracted from tumor tissue that was dissected from a large section of the tumor block corresponding to the respective TMA spot and from nonneoplastic control tissue of the patient. Percentage of tumor cells was at least $50 \%$ within the tumor area analyzed. MSI-high was assigned when at least two of the five markers of the Bethesda-Panel showed instability (e.g., length variation compared to control tissue), and MSI-low was diagnosed if only one of the analyzed loci showed instability. All other cases were considered microsatellite stable (MSS).

\section{Quantification of CD8 Immunostaining}

Digital images of stained slides were acquired using Leica's Aperio VERSA 8 automated microscope. TMA spots were automatically identified and analyzed using the Image Scope 12.3.3 software package (Leica Microsystems; Wetzlar, Germany) according to the following procedure: every TMA slide was scanned at $40 \times$ magnification. Digital images were segmented using the Image Scope brightfield TMA-Tool, and the segmentation was corrected manually. Two Aperio ePathology Image Analysis macros (Leica Microsystems) were adjusted to determine the number of $\mathrm{CD}^{+}$cells in each tissue spot and to measure the corresponding area of each tissue spot. The number of stained cells and the area in square millimeters of each individual spot was used to calculate the density of
$\mathrm{CD}^{+}$stained cells $/ \mathrm{mm}^{2}$ (number of cells per square $\mathrm{mm}$ ). Schematic representation of the workflow to detect CD8 positive cell density is shown in supplementary Fig. 1.

\section{Statistics}

Statistical calculations were performed with JPM 14 software (SAS Institute Inc., NC, USA) and R version 3.5.1 (The $\mathrm{R}$ foundation). ${ }^{32}$ Analysis of variance (ANOVA) was performed to search for associations between the density of CD8 positive cells and tumor phenotype as well as MSI status.

\section{RESULTS}

\section{TMA Screening}

A total of 542 of 597 (91\%) cancers on the TMA were considered interpretable for MMR status, because either an unequivocal loss of staining of at least one of the four examined MMR proteins MLH1, PMS2, MSH2, or MSH6 was seen (MMR deficiency) or unequivocally retained expression of at least $3 \mathrm{MMR}$ proteins without concomitant loss was observed (intact MMR status). Noninterpretable cancers $(n=55 ; 9 \%)$ were due to lack of unequivocal tumor on TMA spots or incomplete MMR results for tumor tissue, defined as retained expression but results available for $\leq 2$ markers only. From the 542 interpretable cancers, 511 showed retained expression for all four MMR proteins (intact MMR status). In 25 cases, one MMR protein was not evaluable (due to lack of tissue or unequivocal tumor tissue on the spot) but the remaining 3 markers were unequivocally retained. These tumors were also considered to have an intact MMR status. For the remaining 6 cancers, the respective TMA spots showed loss of one or two MMR proteins with adequate positive control and were thus considered suspicious for MSI (Table 1). Representative micrographs from TMA screening are shown in Fig. 1.

\section{MSI Validation and Heterogeneity Analysis}

Large section examination confirmed MMR deficiency in four of six suspected cases, all ductal adenocarcinomas, suggesting a prevalence for MSI in pancreatic ductal adenocarcinoma of $0.8 \%$ (4/480). Discrepant MMR status between TMA spots and large sections were always due to heterogeneous staining across the selected tumor blocks including areas where immunostaining was still weakly visible in some stromal cells but not visible in tumor cells. In these cases, the TMA cylinder had unluckily been taken from areas with markedly diminished immunoreactivity. 
All four confirmed MMR-deficient cases demonstrated MSH6 loss. MSH2 was additionally lost in three of these cases. Representative images from large sections are shown in Fig. 2. MSI analysis by PCR revealed MSI-high in three cancers and MSS in the cancer with isolated MSH6 loss (Table 1). A total of 44 large sections were analyzed from 4 patients (6-16 tumor blocks per patient) and revealed completely homogeneous MMR deficiency for each of the 4 identified MMR deficient cancers, including lymph node metastases that were present in 3 patients.

\section{Clinical Evaluation}

Available clinical data revealed that additional cancers with MSI had occurred in two of the four patients with confirmed MMR deficient pancreatic cancer. One of these patients had a metachronous endometrioid endometrial cancer and the other patient a synchronous adenocarcinoma of the colon, both with MSH2 and MSH6 loss. In both cases, the expression loss involved the same MMR proteins as seen in the respective pancreatic cancer. For the other two patients with MMR-deficient pancreatic cancer, no clinical indications for Lynch Syndrome were found.

\section{Density of CD8-positive T Lymphocytes}

Density of CD8-positive cells could be evaluated in 551 of 597 cancers $(92 \%)$ and varied widely from 0 to 2367 cells $/ \mathrm{mm}^{2}$ in 551 interpretable cancers $($ median $=125$; mean $=231$ ). Representative images are shown in Fig. 3 . The density of CD8-positive cells did not significantly vary between pancreatic ductal adenocarcinomas $(n=488$; mean $=221$, median $=122$ ), adenocarcinomas of the ampulla of Vater $(n=54$; mean $=301$, median $=134)$, and acinar cell carcinomas $(n=7$; mean $=256$, median $=50 ; p=0.17)$. The further comparison of the density of CD8-positive cells with tumor phenotype and MMR deficiency was limited to the ductal subset of pancreas cancers. A high density of CD8-positive cells was significantly associated with MMR deficiency. The density of CD8-positive cells was markedly higher in the 4 MMRdeficient cancers $($ mean $=685$, median $=626)$ than in 520 cancers with intact MMR (mean $=227$; median $=124$; $p<0.0001)$. The cancer with unequivocal MMR defect (MSH6 loss) but MSS showed a particularly high CD8 density (958 cells $/ \mathrm{mm}^{2}$; Table 1 ). The density of CD8 positive cells was statistically unrelated to $\mathrm{pT}, \mathrm{pN}, \mathrm{M}$ status, tumor grade (all supplementary Table 1), and tumor diameter $(p=0.4225$, data not shown). 
FIG. 1 TMA spots of one pancreatic ductal adenocarcinoma with MSI associated with protein loss of MSH6 (b), whereas protein expression of MSH2 (a), MLH1 (c), and PMS2 (d) is retained. Original magnifications $15 \mathrm{x}$, spot size $600 \mu \mathrm{m}$
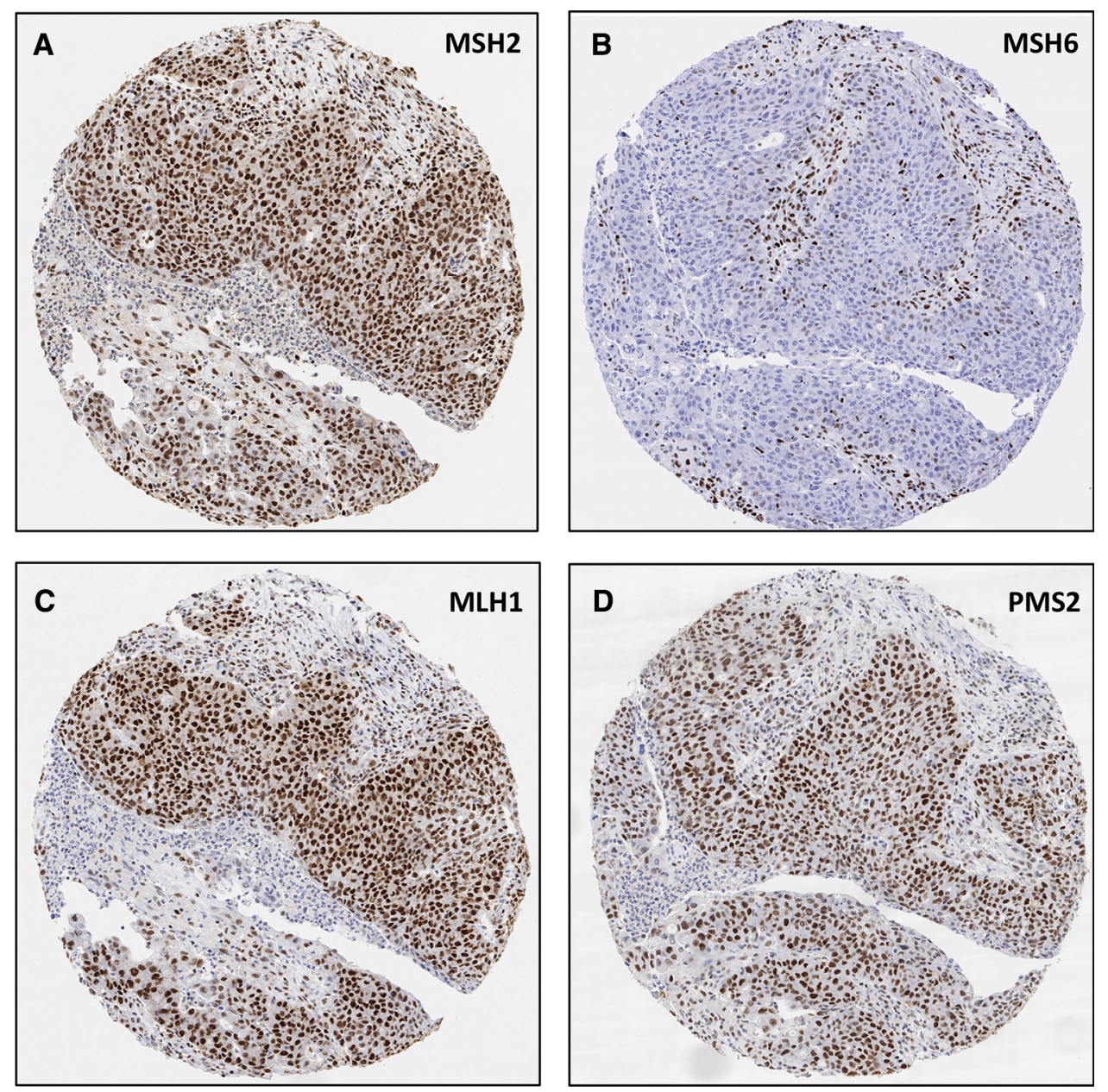

\section{DISCUSSION}

In this study we used a TMA for rapid screening for MMR deficiency in 597 carcinomas of the pancreas. Rare event detection is an ideal application for TMAs. Previous studies have found 42 tumors harboring IDH1 mutations by screening 15,531 prostate cancers $(0.3 \%)$ or 43 tumors exhibiting CD117 overexpression in a cohort of 1654 breast carcinomas $(2.6 \%)$. $^{33,34} \mathrm{It}$ is of note that only four of six cancers that were initially suspected to have MSI were confirmed in a subsequent large section validation. In both cancers that were not confirmed to exhibit MSI, the discrepancies were due to a staining gradient across the large slide, which is typically caused by inhomogeneous tissue fixation. In these cases, the tumor cells completely lacked MMR protein staining in the respective TMA spots while some weak staining was seen in stromal cells. This may reflect somewhat higher expression levels in a subset of stromal cells compared with the cancer cells in these cases.

Our rate of $0.8 \%$ of pancreatic ductal adenocarcinomas having MSI fits well with data from recent studies applying next generation sequencing. Salem et al. analyzed 7000 microsatellite loci in 870 pancreatic cancers by NGS and described $1.1 \%$ of tumors as MSI-high using NGS criteria. ${ }^{35} \mathrm{Hu}$ et al. used a deep sequencing approach to evaluate microsatellites in all exons and selected introns of 341-468 cancer associated genes, followed by PCR and IHC validation and found MSI in $0.8 \%$ of pancreatic cancer. ${ }^{36}$ Findings were more variable in pure IHC studies and early PCR-based investigations using less stringent criteria to define MSI. Earlier IHC studies described MMR deficiency in $0-22 \% .{ }^{22,23}$ Highest rates were reported in two TMA studies describing MMR deficiency in $15 \%$ of 265 and in $22 \%$ of 109 resected pancreatic cancers. ${ }^{22,37}$ Immunostaining issues in case of unevenly fixed tissues, as seen in our study, may serve as an explanation for such high frequencies of undetectable MMR protein in cancers. A large recent IHC-based analysis found MSI in $1.6 \%$ of 445 ductal pancreatic adenocarcinomas, which comes close to the ratio found by us. ${ }^{38}$ Earlier PCR-based studies on unselected cohorts of pancreatic adenocarcinomas described MSI in $0-17 \%$ of cases. ${ }^{24,25}$ The study with the highest fraction of positive cases found MSI in 8 of 46 cases, defining MSI as instability in at least 3 of 8 microsatellite 

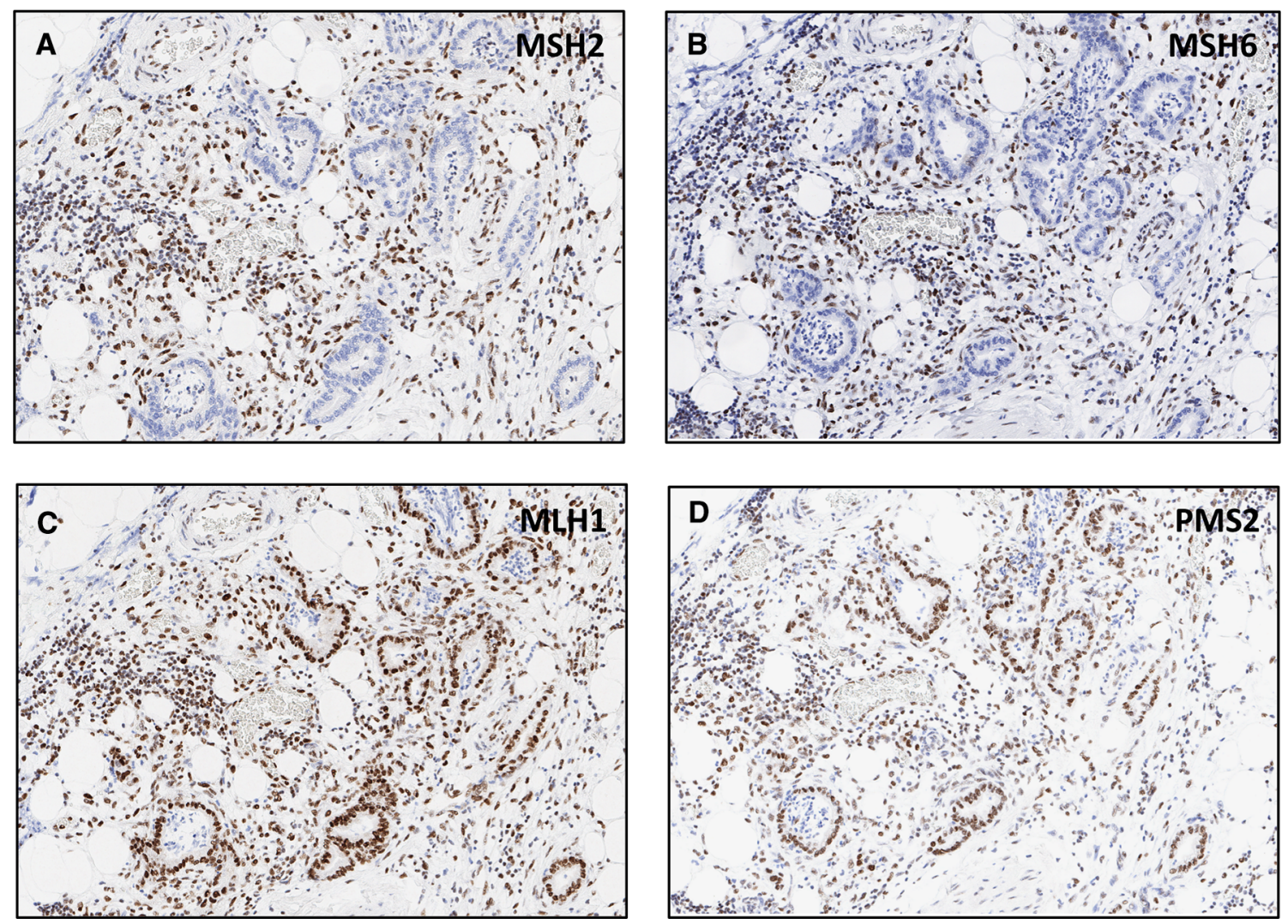

FIG. 2 Protein loss of MSH2 (a) and MSH6 (b) on large sections of one pancreatic ductal adenocarcinoma. MLH1 (c) and PMS2 (d) protein expression is retained in the tumor cells. Staining in stromal cells and inflammatory cells is present as internal control. Original magnification 20x
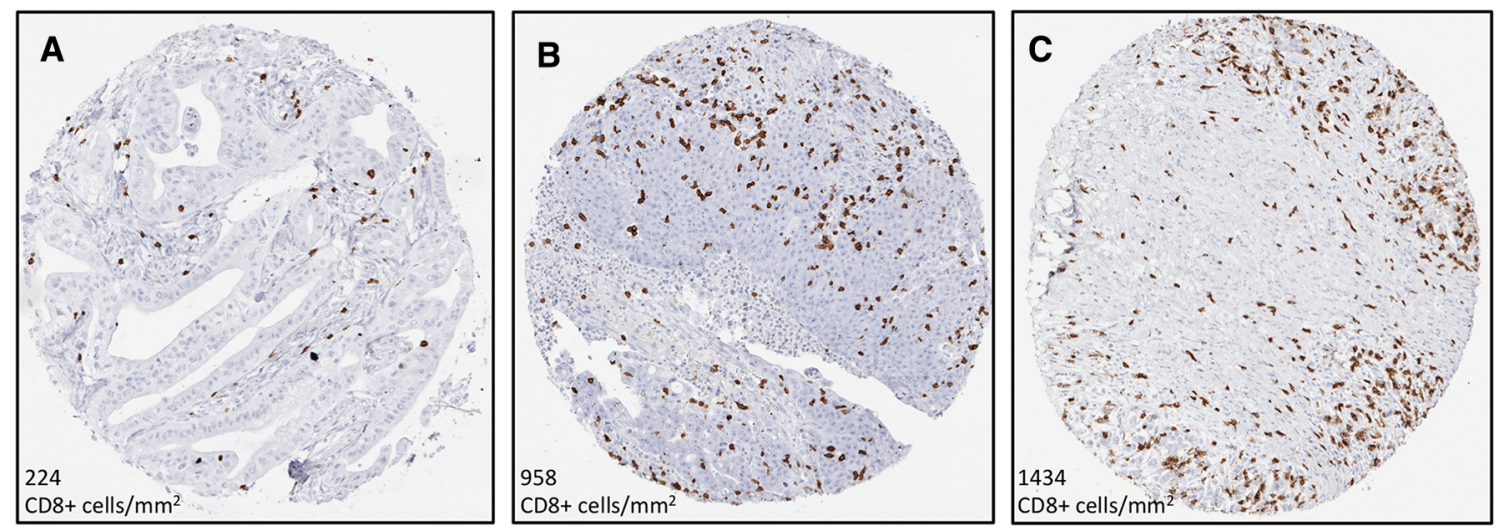

FIG. 3 CD8-positive cell density in pancreatic ductal adenocarcinoma with intact MMR (a), with isolated protein loss of MSH6 associated with MSS in PCR-analysis (b), and with protein loss of MSH2 and MSH6 associated with MSI-high (c)

loci analyzed. ${ }^{25}$ High rates of MSI $(22 \%)$ also have been described in a selected cohort of 18 medullary pancreatic carcinomas. $^{39}$

Evaluation of the MMR status throughout all available cancer-containing tumor blocks revealed homogeneous MMR protein loss throughout primary tumor and-if present-nodal metastases in all four ductal pancreatic adenocarcinomas with confirmed MSI. This fits with our previous observations of high homogeneity of MSI in prostate, bladder, ovarian, and neuroendocrine colorectal cancer (unpublished data). ${ }^{40}$ Overall, these data may suggest that MMR inactivation generally occurs early in tumorigenesis. Clinical history was suggestive of Lynch Syndrome in two of the four patients with MMR deficient pancreatic cancer as a metachronous MSI tumor with identical MMR pattern was reported in each case, presenting an explanation for the early MMR inactivation. Irrespective of its underlying cause (hereditary versus 
sporadic), homogeneity of MMR deficiency reduces the risk that microsatellite status obtained from small biopsies may not be representative for the entire cancer mass. Intratumoral heterogeneity is a potential strong confounder for individualized therapies, especially in pancreatic cancers that are not amenable to surgery.

Contemporary studies using state of the art technology usually reported MMR protein defects and MSI in about $0.5-3 \%$ of cancers, irrespective of the cancer type analyzed. ${ }^{14}$ Accordingly, the absence of detectable MMR protein loss in any of the 55 interpretable adenocarcinomas of the ampulla of Vater or 7 acinar cell carcinomas does not suggest absence of MSI in these tumor entities. In fact, MSI has previously been reported for both tumor entities in studies employing IHC. Agaram et al. found a MMR protein loss in 5 of 36 acinar cell carcinomas (14\%), and Liu et al. reported MMR deficiency in 6 of 54 ampullary carcinomas $(11 \%){ }^{41,42}$ Our results may suggest lower rates in these tumor types.

All four pancreatic ductal adenocarcinomas with MMR deficiency in our study exhibited protein loss of MSH6, which was accompanied by protein loss of MSH2 in three cancers and was isolated in one cancer. The functional role of MSH6 depends on heterodimerization with MSH2 (MutSalpha). ${ }^{43}$ Inactivation of MSH2, for instance due to proteasome-dependent degradation described for several disease-causing $\mathrm{MSH} 2$ mutations, results in concomitant MSH6 inactivation, because failure of heterodimerization causes rapid MSH6 degradation. ${ }^{44,45}$ In contrast, when MSH6 is inactivated, MSH2 is preserved due to increase of MSH3 by enhanced transcription and protein stability, facilitating alternative heterodimerization of $\mathrm{MSH} 2$ with MSH3 (MutSbeta). ${ }^{46}$ Accordingly, isolated MSH6 loss may be due to MSH6 inactivation while loss of both MSH6 and $\mathrm{MSH} 2$ may be attributable to MSH2 inactivation. Combined loss of MSH2 and MSH6 is rather infrequent in colorectal, endometrial, and gastric cancer, the tumor types with highest prevalence of MSI. ${ }^{47,48}$ In these entities, the vast majority of sporadic tumors with MSI are associated with MLH1 inactivation due to promotor hypermethylation. Consequently, MSI associated with loss of MSH2 and MSH6 is regarded as suspicious for a hereditary tumor origin in these cancer types. ${ }^{49-51}$ This may also hold true for a fraction of pancreatic cancers. In a recent NGS analysis of 53 hereditary pancreatic cancers without any known predisposition gene, one MSH2 and MSH6 germline mutation was described each. ${ }^{52}$ All 7 tumors with MSI among 833 pancreatic cancers $(0.8 \%)$ identified in a recent study by $\mathrm{Hu}$ et al. using NGS sequencing were found to harbor germline mutations in the MMR genes. ${ }^{36}$ The concept of a possible germline involvement in pancreatic cancers with MSH6 and/or MSH2 expression loss is also supported by our findings. Two of our four patients with MSI pancreatic cancers had metachronous carcinomas with identical MMR protein loss.

It is of note that the tumor with an isolated but clear-cut loss of MSH6 expression was microsatellite stable in our PCR analysis. This is not surprising as a $100 \%$ concordance between MSI and MMR analysis is usually not found. ${ }^{53,54}$ Bartley et al. reported 13 discordant tumors among 591 colorectal carcinomas $(2.2 \%) .{ }^{53}$ Discordance rate was even higher (5.5\%) among 1.119 carcinomas from patients meeting clinical screening criteria (Amsterdam II or classical Bethesda guidelines) for Lynch Syndrome in a study by Engel et al. and Chapusot et al., which found 8 discordant cases among 100 sporadic right-sided colon cancers. ${ }^{27,55}$ Importantly, most discordant cases reported in the literature relate to MSI-high tumors lacking evidence for MMR deficiency by IHC. ${ }^{53}$ First, inactivating MMR mutations that escape detection by IHC-typically nonsense/missense mutations impairing protein function but retained antigenicity of the altered protein-may explain this. ${ }^{55}$ Second, the MMR system has much more players than MLH1, PMS2, MLH2, and MHS6. Inactivation of other MMR proteins, such as MSH3, PMS1, or EPCAM, may cause MSI without evidence of MMR deficiency by IHC. Discordant results characterized by IHC-detected MMR deficiency without MSI in PCR are generally rather rare but are well characterized for inactivating MSH6 mutations. ${ }^{56} \mathrm{MSH} 3$ can partly compensate inactivated MLH6. However, in particular, MSH2/MSH3 heterodimers do not repair single base excisions as effectively as does the MSH2/MSH6 heterodimer. ${ }^{57}$ As the "Bethesda Panel"-used for defining MSI in this study-merely incorporates two mononucleotide repeats, MSI driven by MSH6 inactivation can be missed by PCR. In fact, no instability was found among any of the five repeat loci in the MSI tumor with isolated MSH6 loss in our study. Moreover, the "Bethesda Panel" (i.e., the selection of five mono- and dinucleotide repeats from the myriad of microsatellite loci throughout the genome) was developed based on data from MSI in colorectal cancers. ${ }^{58}$ Individual microsatellite loci may not be equally often affected by instability in different tissues and cell types, which relates to the observation that the likelihood for a frameshift depends on the transcriptional activity of the respective genomic region. ${ }^{59,60}$ Therefore, it is conceivable that the "Bethesda Panel" may not be universally suited for MSI detection across different tumor types.

Indirect support for true MMR deficiency in the identified MSS tumor with MSH6 protein loss comes from its strikingly high density of intratumoral CD8 positive lymphocytes $\left(958\right.$ cells $/ \mathrm{mm}^{2}$ ). The significant association between MMR deficiency and high number of intratumoral CD8-positive lymphocytes found for pancreatic cancer in 
this study is consistent with earlier data from colorectal, endometrial and stomach cancer. ${ }^{19-21,61}$ Elevated lymphocyte counts may represent evidence for immunogenic events having occurred in a cancer, a feature that is believed to be essential for successful therapy with immune checkpoint inhibitors. The significantly higher CD8 density in MMR deficient compared with MMR intact pancreatic cancers may thus provide an additional hint towards a potential utility of immune checkpoint inhibitors in pancreatic cancers as suggested by the successful treatment of several cancers in a site-agnostic clinical trial. ${ }^{6}$

Paralleling the advances in individual tumor therapy in modern oncology and the site-agnostic approval of recent drugs, there is a rapid increase of tumor testing in molecular pathology to identify "druggable" targets and a practical screening strategy to decide which tumor to test for certain alterations is desirable. Is it reasonable to test every pancreatic cancer for MMR deficiency and/or MSI, although the expected rate of MSI is "only" around $1 \%$ ? In contrast to other widely performed predictive tests-like fluorescence in situ-hybridization for ROS1 or ALK fusion oncogenes in non-small cell lung cancer with reported frequencies only slightly higher (2-5\%) - MMR IHC is fast and relatively inexpensive. ${ }^{62}$ Considering also the compelling response rates for immune checkpoint inhibitors in pancreatic cancers with MMR deficiency/MSI, including complete radiologic response in individual tumors, testing for MMR deficiency/ MSI in pancreatic cancer may generally be considered and is encouraged in locally advanced/metastatic cancers by a recent NCCN guidelines update. ${ }^{6,63}$ Furthermore, detection of MMR deficiency/MSI can help to identify Lynch Syndrome patients and the evolving scientific interest in MSI has now made clear that MSI cancers due to germline mutations of MMR genes are not limited to colorectal and endometrial cancer as around half of all germline MMR mutations do occur in other tumor types, including pancreatic cancer. ${ }^{15}$ Parallel sequencing (NGS-based) methods to detect MSI have recently been developed, which may simplify molecular testing algorithms in cancer patients in the future, at least for tumor types where NGS is routinely applied to search for therapeutic targets. ${ }^{64,65}$

It is a limitation to this study that follow-up data were unavailable from our patients. The clinical impact of MSI and the density of CD8 positive cells thus cannot be finally judged based on our data. The absence of a significant association of the density of CD8-positive cells and pT, $\mathrm{pN}, \mathrm{M}$ status, tumor grade, and tumor diameter does not suggest a pivotal clinical relevance of the CD8 density, at least in the absence of therapy regimens targeting the immune system. Several studies on cohorts from 86 to 214 patients with pancreatic cancer have, in contrast, suggested an association between the fraction of CD8 positive lymphocytes or neutrophils with tumor aggressiveness or clinical outcome of pancreatic cancer. ${ }^{66-68}$ However, spatial variance of CD8-positive cells-typically between central and peripheral tumor areas-is quite prevalent in pancreatic cancer and may confound analyses when information on the precise localization of the analyzed tumor area is not available. ${ }^{66}$ TMA-based studies-at least when TMA construction was not focused on tumor compartments as in our study-may underrepresent the invasive margin that has been shown to confer a prognostic role for CD8-positive cells in pancreatic cancer in particular. ${ }^{68}$ Furthermore, pancreatic cancer possess a unique microenvironment characterized by a dense desmoplastic stromal reaction that may enhance regional variability of lymphocytic density. ${ }^{69}$ For instance, CD8-positive cells at the invasive margin were significantly associated with overall survival in contrast to the tumor core. ${ }^{70}$ In that study, Tahkola et al. already used two TMA cores from each the tumor center and the invasive margin and only counted the higher score for statistical analyses to account for regional variation of $\mathrm{CD} 8$ positive cell density. ${ }^{70}$

\section{CONCLUSIONS}

MSI identified by detecting MMR protein loss with IHC occurs in approximately $1 \%$ of pancreatic adenocarcinomas. The complete homogeneity seen in all four cancers with MSI suggests that MMR deficiency often is an early event in affected tumors. Small tissue probes as obtainable by biopsy or even aspiration cytology thus may be sufficient to determine a MSI status representative for the entire cancer mass.

Electronic supplementary material The online version of this article (https://doi.org/10.1245/s10434-020-08209-y) contains supplementary material, which is available to authorized users.

ACKNOWLEDGMENTS Open Access funding provided by Projekt DEAL. The author thank Inge Brandt, Sünje Seemann, Melanie Witt, and Maren Eisenberg for excellent technical assistance. Mrs. Devita Irene Putri contributed significantly to this study with results from her medical thesis.

DISCLOSURES The authors declare no conflicts of interst.

OPEN ACCESS This article is licensed under a Creative Commons Attribution 4.0 International License, which permits use, sharing, adaptation, distribution and reproduction in any medium or format, as long as you give appropriate credit to the original author(s) and the source, provide a link to the Creative Commons licence, and indicate if changes were made. The images or other third party material in this article are included in the article's Creative Commons licence, unless indicated otherwise in a credit line to the material. If material is not included in the article's Creative Commons licence and your intended use is not permitted by statutory regulation or exceeds the permitted use, you will need to obtain permission directly from the copyright holder. To view a copy of this licence, visit http://creativecommons. org/licenses/by/4.0/. 


\section{REFERENCES}

1. Siegel RL, Miller KD, Jemal A. Cancer statistics, 2018. $C A$ Cancer J Clin. 2018;68(1):7-30.

2. Brahmer JR, Tykodi SS, Chow LQ, et al. Safety and activity of anti-PD-L1 antibody in patients with advanced cancer. $N$ Engl $J$ Med. 2012;366(26):2455-65.

3. Patnaik A, Kang SP, Rasco D, et al. Phase I study of pembrolizumab (MK-3475; Anti-PD-1 Monoclonal Antibody) in Patients with advanced solid tumors. Clin Cancer Res. 2015;21(19):4286-93.

4. Le DT, Uram JN, Wang H, et al. PD-1 blockade in tumors with mismatch-repair deficiency. N Engl J Med. 2015;372(26):25092520.

5. Lemery S, Keegan P, Pazdur R. First FDA approval agnostic of cancer site-when a biomarker defines the indication. $N$ Engl $J$ Med. 2017:377(15):1409-12.

6. Le DT, Durham JN, Smith KN, et al. Mismatch repair deficiency predicts response of solid tumors to PD-1 blockade. Science. 2017;357(6349):409-13.

7. Backes FJ, Leon ME, Ivanov I, et al. Prospective evaluation of DNA mismatch repair protein expression in primary endometrial cancer. Gynecol Oncol. 2009;114(3):486-90.

8. Joehlin-Price AS, Perrino CM, Stephens J, et al. Mismatch repair protein expression in 1049 endometrial carcinomas, associations with body mass index, and other clinicopathologic variables. Gynecol Oncol. 2014;133(1):43-7.

9. Hause RJ, Pritchard CC, Shendure J, Salipante SJ. Classification and characterization of microsatellite instability across 18 cancer types. Nat Med. 2016;22(11):1342-50.

10. Ashktorab H, Ahuja S, Kannan L, et al. A meta-analysis of MSI frequency and race in colorectal cancer. Oncotarget. 2016;7(23):34546-57.

11. Kim JY, Shin NR, Kim A, et al. Microsatellite instability status in gastric cancer: a reappraisal of its clinical significance and relationship with mucin phenotypes. Korean $J$ Pathol. 2013;47(1):28-35.

12. Cancer Genome Atlas Research N. Comprehensive molecular characterization of gastric adenocarcinoma. Nature. 2014;513(7517):202-9.

13. Mathiak M, Warneke VS, Behrens HM, et al. Clinicopathologic characteristics of microsatellite instable gastric carcinomas revisited: urgent need for standardization. Appl Immunohistochem Mol Morphol. 2017;25(1):12-24.

14. Bonneville R, Krook MA, Kautto EA, et al. Landscape of microsatellite instability across 39 cancer types. JCO Precis Oncol. 2017;2017.

15. Latham A, Srinivasan P, Kemel Y, et al. Microsatellite Instability Is Associated With the Presence of Lynch Syndrome Pan-Cancer. J Clin Oncol. 2019;37(4):286-95.

16. Santoiemma PP, Powell DJ, Jr. Tumor infiltrating lymphocytes in ovarian cancer. Cancer Biol Ther. 2015;16(6):807-20.

17. Andersen R, Donia M, Westergaard MC, Pedersen M, Hansen M, Svane IM. Tumor infiltrating lymphocyte therapy for ovarian cancer and renal cell carcinoma. Hum Vaccin Immunother. 2015;11(12):2790-5.

18. Mahmoud SM, Paish EC, Powe DG, et al. Tumor-infiltrating CD8 + lymphocytes predict clinical outcome in breast cancer. $J$ Clin Oncol. 2011;29(15):1949-55.

19. Smyrk TC, Watson P, Kaul K, Lynch HT. Tumor-infiltrating lymphocytes are a marker for microsatellite instability in colorectal carcinoma. Cancer. 2001;91(12):2417-22.

20. Buckowitz A, Knaebel HP, Benner A, et al. Microsatellite instability in colorectal cancer is associated with local lymphocyte infiltration and low frequency of distant metastases. Br J Cancer. 2005;92(9):1746-53.

21. Suemori $\mathrm{T}$, Susumu $\mathrm{N}$, Iwata $\mathrm{T}$, et al. Intratumoral CD8 + lymphocyte infiltration as a prognostic factor and its relationship with cyclooxygenase 2 expression and microsatellite instability in endometrial cancer. Int $J$ Gynecol Cancer. 2015;25(7):1165-72.

22. Eatrides JM, Coppola D, Al Diffalha S, Kim RD, Springett GM, Mahipal A. Microsatellite instability in pancreatic cancer. J Clin Oncol. 2016 2016;34(15_suppl, e15753).

23. Tomaszewska R, Okon K, Stachura J. Expression of the DNA mismatch repair proteins (hMLH1 and hMSH2) in infiltrating pancreatic cancer and its relation to some phenotypic features. Pol J Pathol. 2003;54(1):31-7.

24. Ghimenti C, Tannergard P, Wahlberg S, et al. Microsatellite instability and mismatch repair gene inactivation in sporadic pancreatic and colon tumours. Br J Cancer. 1999;80(1-2):11-6.

25. Nakata B, Wang YQ, Yashiro $M$, et al. Prognostic value of microsatellite instability in resectable pancreatic cancer. Clin Cancer Res. 2002;8(8):2536-40.

26. Joost $P$, Veurink N, Holck $S$, et al. Heterogenous mismatch-repair status in colorectal cancer. Diagn Pathol. 2014;9:126.

27. Chapusot C, Martin L, Bouvier AM, et al. Microsatellite instability and intratumoural heterogeneity in 100 right-sided sporadic colon carcinomas. Br J Cancer. 2002;87(4):400-4.

28. Watson N, Grieu F, Morris M, et al. Heterogeneous staining for mismatch repair proteins during population-based prescreening for hereditary nonpolyposis colorectal cancer. J Mol Diagn. Sep 2007;9(4):472-8.

29. Watkins JC, Nucci MR, Ritterhouse LL, Howitt BE, Sholl LM. Unusual mismatch repair immunohistochemical patterns in endometrial carcinoma. Am J Surg Pathol. 2016;40(7):909-16.

30. Pai RK, Plesec TP, Abdul-Karim FW, et al. Abrupt loss of MLH1 and PMS2 expression in endometrial carcinoma: molecular and morphologic analysis of 6 cases. Am $J$ Surg Pathol. 2015;39(7):993-9.

31. Kononen J, Bubendorf L, Kallioniemi A, et al. Tissue microarrays for high-throughput molecular profiling of tumor specimens. Nat Med. 1998;4(7):844-7.

32. R-Core-Team. R: A language and environment for statistical computing. $R$ Foundation for Statistical Computing, Vienna, Austria.2018.

33. Hinsch A, Brolund M, Hube-Magg C, et al. Immunohistochemically detected IDH1 $(\mathrm{R} 132 \mathrm{H})$ mutation is rare and mostly heterogeneous in prostate cancer. World $J$ Urol. 2018;36(6):877-82.

34. Simon R, Panussis S, Maurer R, et al. KIT (CD117)-positive breast cancers are infrequent and lack KIT gene mutations. Clin Cancer Res. 2004;10(1 Pt 1):178-183.

35. Salem ME, Puccini A, Grothey A, et al. Landscape of tumor mutation load, mismatch repair deficiency, and pd-11 expression in a large patient cohort of gastrointestinal cancers. Mol Cancer Res. 2018;16(5):805-12.

36. Hu ZI, Shia J, Stadler ZK, et al. Evaluating Mismatch Repair Deficiency in Pancreatic Adenocarcinoma: Challenges and Recommendations. Clin Cancer Res. 2018;24(6):1326-36.

37. Riazy M, Kalloger SE, Sheffield BS, et al. Mismatch repair status may predict response to adjuvant chemotherapy in resectable pancreatic ductal adenocarcinoma. Mod Pathol. 2015;28(10):1383-9.

38. Lupinacci RM, Goloudina A, Buhard O, et al. Prevalence of microsatellite instability in intraductal papillary mucinous neoplasms of the pancreas. Gastroenterology. 2018;154(4):1061-5.

39. Wilentz RE, Goggins M, Redston M, et al. Genetic, immunohistochemical, and clinical features of medullary carcinoma of 
the pancreas: A newly described and characterized entity. Am J Pathol. 2000;156(5):1641-51.

40. Fraune C, Simon R, Hoflmayer D, et al. High homogeneity of mismatch repair deficiency in advanced prostate cancer. Virchows Arch. 2019.

41. Liu W, Shia J, Gonen M, Lowery MA, O’Reilly EM, Klimstra DS. DNA mismatch repair abnormalities in acinar cell carcinoma of the pancreas: frequency and clinical significance. Pancreas. 2014;43(8):1264-70.

42. Agaram NP, Shia J, Tang LH, Klimstra DS. DNA mismatch repair deficiency in ampullary carcinoma: a morphologic and immunohistochemical study of 54 cases. Am J Clin Pathol. 2010;133(5):772-80.

43. Acharya S, Wilson T, Gradia S, et al. hMSH2 forms specific mispair-binding complexes with hMSH3 and hMSH6. Proc Natl Acad Sci U S A. 1996;93(24):13629-34.

44. Nielsen SV, Stein A, Dinitzen AB, et al. Predicting the impact of Lynch syndrome-causing missense mutations from structural calculations. PLoS Genet. 2017;13(4):e1006739.

45. Marra G, Iaccarino I, Lettieri T, Roscilli G, Delmastro P, Jiricny J. Mismatch repair deficiency associated with overexpression of the MSH3 gene. Proc Natl Acad Sci $U$ S A. 1998;95(15):8568-73.

46. Chang DK, Ricciardiello L, Goel A, Chang CL, Boland CR. Steady-state regulation of the human DNA mismatch repair system. J Biol Chem. 2000;275(24):18424-31.

47. Cho J, Kang SY, Kim KM. MMR protein immunohistochemistry and microsatellite instability in gastric cancers. Pathology. 2019;51(1):110-3.

48. Lee HJ, Jang YJ, Lee EJ, et al. The significance of mismatch repair genes in gastric cancer. $J$ Cancer Res Ther. 2013;9(1):80-3.

49. Metcalf AM, Spurdle AB. Endometrial tumour BRAF mutations and MLH1 promoter methylation as predictors of germline mismatch repair gene mutation status: a literature review. Fam Cancer.2014;13(1):1-12.

50. Mills AM, Liou S, Ford JM, Berek JS, Pai RK, Longacre TA. Lynch syndrome screening should be considered for all patients with newly diagnosed endometrial cancer. Am J Surg Pathol. 2014;38(11):1501-9.

51. Cunningham JM, Christensen ER, Tester DJ, et al. Hypermethylation of the hMLH1 promoter in colon cancer with microsatellite instability. Cancer Res. Aug 1 1998;58(15):3455-60.

52. Slavin TP, Neuhausen SL, Nehoray B, et al. The spectrum of genetic variants in hereditary pancreatic cancer includes Fanconi anemia genes. Fam Cancer. 2018;17(2):235-45.

53. Bartley AN, Luthra R, Saraiya DS, Urbauer DL, Broaddus RR. Identification of cancer patients with Lynch syndrome: clinically significant discordances and problems in tissue-based mismatch repair testing. Cancer Prev Res (Phila). 2012;5(2):320-7.

54. Cicek MS, Lindor NM, Gallinger S, et al. Quality assessment and correlation of microsatellite instability and immunohistochemical markers among population- and clinic-based colorectal tumors results from the Colon Cancer Family Registry. J Mol Diagn. May 2011;13(3):271-81.

55. Engel C, Forberg J, Holinski-Feder E, et al. Novel strategy for optimal sequential application of clinical criteria, immunohistochemistry and microsatellite analysis in the diagnosis of hereditary nonpolyposis colorectal cancer. Int $J$ Cancer. 2006;118(1):115-22.

56. Berends MJ, Wu Y, Sijmons RH, et al. Molecular and clinical characteristics of MSH6 variants: an analysis of 25 index carriers of a germline variant. Am J Hum Genet. Jan 2002;70(1):26-37.

57. Umar A, Risinger JI, Glaab WE, Tindall KR, Barrett JC, Kunkel TA. Functional overlap in mismatch repair by human MSH3 and MSH6. Genetics. 1998;148(4):1637-46.

58. Boland CR, Thibodeau SN, Hamilton SR, et al. A national cancer institute workshop on microsatellite instability for cancer detection and familial predisposition: development of international criteria for the determination of microsatellite instability in colorectal cancer. Cancer Res. 1998;58(22):5248-57.

59. Schuster-Bockler B, Lehner B. Chromatin organization is a major influence on regional mutation rates in human cancer cells. Nature. 2012;488(7412):504-7.

60. Supek F, Lehner B. Differential DNA mismatch repair underlies mutation rate variation across the human genome. Nature. 2015;521(7550):81-4.

61. De Rosa S, Sahnane N, Tibiletti MG, et al. EBV(+) and MSI Gastric Cancers Harbor High PD-L1/PD-1 Expression and High CD8(+) Intratumoral Lymphocytes. Cancers (Basel). 2018;10(4).

62. IASLC. IASLC Atlas of ALK and ROS1 testing in Lung Cancer Editorial Rx Press; Second Edition, 2016.

63. NCCN Flash Update: Pancreatic Adenocarcinoma. https://www. nccn.org/about/news/ebulletin/ebulletindetail.aspx?ebulletinid=1 193. Accessed 12 Dec 2019.

64. Vanderwalde A, Spetzler D, Xiao N, Gatalica Z, Marshall J. Microsatellite instability status determined by next-generation sequencing and compared with PD-L1 and tumor mutational burden in 11,348 patients. Cancer Med. 2018;7(3):746-56.

65. Salipante SJ, Scroggins SM, Hampel HL, Turner EH, Pritchard CC. Microsatellite instability detection by next generation sequencing. Clin Chem. 2014;60(9):1192-9.

66. Masugi Y, Abe T, Ueno A, et al. Characterization of spatial distribution of tumor-infiltrating $\mathrm{CD} 8(+) \mathrm{T}$ cells refines their prognostic utility for pancreatic cancer survival. Mod Pathol. 2019;32(10):1495-507.

67. Hou YC, Chao YJ, Hsieh MH, Tung HL, Wang HC, Shan YS. Low CD8(+) $\mathrm{t}$ cell infiltration and high pd-11 expression are associated with level of cd44(+)/cd133(+) cancer stem cells and predict an unfavorable prognosis in pancreatic cancer. Cancers (Basel). 2019;11(4).

68. Miksch RC, Schoenberg MB, Weniger M, et al. Prognostic Impact of Tumor-Infiltrating Lymphocytes and Neutrophils on Survival of Patients with Upfront Resection of Pancreatic Cancer. Cancers (Basel). 2019;11(1).

69. Feig C, Gopinathan A, Neesse A, Chan DS, Cook N, Tuveson DA. The pancreas cancer microenvironment. Clin Cancer Res. 2012;18(16):4266-76.

70. Tahkola K, Mecklin JP, Wirta EV, et al. High immune cell score predicts improved survival in pancreatic cancer. Virchows Arch. 2018;472(4):653-65.

Publisher's Note Springer Nature remains neutral with regard to jurisdictional claims in published maps and institutional affiliations. 